\title{
Evidence for effective interventions to reduce mental health-related stigma and discrimination in the medium and long term: systematic review
}

\author{
N. Mehta, ${ }^{*}$ S. Clement, ${ }^{*}$ E. Marcus, A.-C. Stona, N. Bezborodovs, S. Evans-Lacko, J. Palacios,
}

M. Docherty, E. Barley, D. Rose, M. Koschorke, R. Shidhaye, C. Henderson and G. Thornicroft

\section{Background}

Most research on interventions to counter stigma and discrimination has focused on short-term outcomes and has been conducted in high-income settings.

\section{Aims}

To synthesise what is known globally about effective interventions to reduce mental illness-based stigma and discrimination, in relation first to effectiveness in the medium and long term (minimum 4 weeks), and second to interventions in low- and middle-income countries (LMICS).

\section{Method}

We searched six databases from 1980 to 2013 and conducted a multi-language Google search for quantitative studies addressing the research questions. Effect sizes were calculated from eligible studies where possible, and narrative syntheses conducted. Subgroup analysis compared interventions with and without social contact

\section{Results}

Eighty studies $(n=422653)$ were included in the review. For studies with medium or long-term follow-up (72, of which 21 had calculable effect sizes) median standardised mean differences were 0.54 for knowledge and -0.26 for stigmatising attitudes. Those containing social contact (direct or indirect) were not more effective than those without. The 11 LMIC studies were all from middle-income countries. Effect sizes were rarely calculable for behavioural outcomes or in LMIC studies.

\section{Conclusions}

There is modest evidence for the effectiveness of anti-stigma interventions beyond 4 weeks follow-up in terms of increasing knowledge and reducing stigmatising attitudes. Evidence does not support the view that social contact is the more effective type of intervention for improving attitudes in the medium to long term. Methodologically strong research is needed on which to base decisions on investment in stigma-reducing interventions.

\section{Declaration of interest}

None.

\section{Copyright and usage}

(C) The Royal College of Psychiatrists 2015. This is an open access article distributed under the terms of the Creative commons Attribution (CC BY) licence.
Since Goffman's seminal work on stigma, ${ }^{1}$ research in this field has steadily grown, ${ }^{2}$ although most work consists of surveys among the general public about attitudes towards people with mental illness, ${ }^{3-6}$ and much less is known about effective interventions to reduce stigma, ${ }^{6}$ or about stigma in low- and middle-income countries (LMICs). ${ }^{7-10}$ To better understand the evidence base on interventions to reduce mental illness-related stigma and discrimination, we identified eight existing systematic reviews on this topic. ${ }^{11-18}$ The reviews varied widely in their methods and foci. There was considerable methodological and clinical heterogeneity in the included studies, and consequently metaanalysis was only undertaken in one review, ${ }^{11}$ and for small subgroups in two others. ${ }^{12,13}$ Four reviews presented data or commented on the overall pattern of effect sizes, ${ }^{11-14}$ and in each of these the interventions had small to moderate effects, using Cohen's interpretation. ${ }^{19}$ There was clearest consensus that the interventions containing social contact and first-person narratives were more effective than others. ${ }^{11,13,15,16}$ Two of the reviews explored moderators of effects to understand which types of contact work best, ${ }^{11,13}$ but there is a need for more research in this area. Two reviews indicated that some interventions have the potential to worsen stigma. ${ }^{13,17}$ Most of the reviews were critical of the methodological quality of the included studies, ${ }^{12-15,18}$ commenting in particular on the need for more randomised controlled trials (RCTs) and robust methods generally; the use

*Joint first authors. of unvalidated measures; and the relative lack of follow-up beyond the immediate post-intervention period. Other study limitations noted were the use of convenience samples, ${ }^{13,15,17}$ small sample sizes, ${ }^{14}$ or inappropriate outcome measures. ${ }^{14,15}$ Some reviews highlighted the poor quality of the interventions, which were sometimes delivered without training, manualisation or fidelity checks, ${ }^{11}$ and interventions often lacked a theoretical underpinning and developmental research. ${ }^{13,14}$ In all except one review, which was restricted to studies in Iran, ${ }^{12}$ interventions taking place in LMICs were a small minority or did not feature. From this scoping of existing systematic reviews we concluded that there was a need for a further systematic review to synthesise the evidence on two key issues: effectiveness in the longer term and in LMIC contexts. Consequently this systematic review aimed to assess the effectiveness of interventions (of any type with any target population), compared with inactive or baseline comparators, in reducing mental health-related stigma (knowledge, attitudes and behaviour) using any quantitative study design, addressing specifically the evidence for medium- and long-term effectiveness (research question 1) and the effectiveness of interventions in LMICs (research question 2).

\section{Method}

Studies were included if they described any type of intervention with a stated aim of changing mental health-related stigma or with an implied aim of changing stigma as indicated by the inclusion of 
at least one of the following core stigma-related outcomes: stigma (any), prejudice (attitudes and related outcomes), discrimination, internalised/self-stigma or public mental health awareness/literacy. Intervention studies were included if they related to functional mental illnesses; interventions solely about, or delivered to, populations with dementia, substance misuse, intellectal disabilities or developmental disorders were excluded from this review. We included all quantitative study designs, including RCTs, controlled and uncontrolled pre-post studies, crossover studies, cohort studies and longitudinal panel studies. Studies with more than one intervention group were included. To be eligible, studies needed to report a comparison with a control group (including treatment as usual, best available current treatment or an active control, to control for non-specific effects of the intervention) or a baseline comparator. Studies needed to include at least one stigma outcome which we categorised as related to knowledge, attitudes (prejudice, self- stigma, self-esteem) or behaviour (discrimination, stigmacoping). To be eligible studies also had to address one of our two research questions: to have at least one follow-up point at least 4 weeks after the intervention was completed (to reflect the importance of medium- and longer-term outcomes relevant to stigma, as this is often described by people with mental illness as a long-term challenge); or for the intervention to be carried out in an LMIC setting. Eligibility criteria are shown in the Appendix.

\section{Information sources and search strategy}

We identified studies by searching electronic databases, handchecking reference lists of reviews and consulting with experts in the working group with knowledge of papers in press. We searched the following databases between 25 January 2013 and 8 February 2013: Medline, PsycINFO, the Cochrane Library, the Cumulative Index to Nursing and Allied Health Literature (CINAHL), the Social Science Citation Index (SSCI) and Global Health. In addition we conducted a Google advanced search focusing on LMICs (see Fig. 1 for details). The Google search was warranted in the light of the limited amount of stigma research in LMICs, but was precluded for our first research question as research from high-income countries is more likely to be found through a standard systematic review search. A search strategy was developed by consensus among authors (N.M., S.C., E.B. and M.D.) using both MeSH and text word searching. We searched using the format 'Stigma' OR 'Discrimination' OR synonyms AND 'mental health' OR 'mental disorders' OR synonyms AND 'Intervention Studies' OR synonyms. The full Medline search strategy is shown in online Table DS1. The search was restricted to results between 1980 and 2013 and studies on human beings, but was not limited by language. The decision to start the search at 1980 was a pragmatic one based on our examination of the existing reviews which revealed that the vast majority of stigma intervention research commenced after 1980. Relevant non-English language papers were read by fluent native language speakers in French and Spanish according to the linguistic skills available to members of the review team. Potentially relevant papers in many important languages, including Chinese, were therefore excluded from the review. Systematic and non-systematic reviews were identified during the search and the reference lists of these studies were hand-checked.

\section{Study selection and data collection}

All identified titles and abstracts were screened by two researchers. Because of the large number of search hits, two researchers screened $5 \%$ of abstracts together. As good agreement (>95\%) was achieved, the remainder were divided between the two researchers and study selection conducted by one researcher for each half. Where the researcher was unclear as to whether a paper should be included, the paper was discussed in consensus meetings. Two review authors extracted data from included studies for all parts of the systematic review, with queries resolved by discussion and consensus.

\section{Statistical analysis}

Outcomes for the studies included were reported using both scales and individual items, although for the effect size calculations were restricted to scale data for knowledge and attitudes. We classified all reported stigma outcomes into the categories of 'knowledge', 'attitudes' or 'behaviour'. Differences between intervention group and control group at follow-up were our main focus for the quantitative review. Effect sizes, standardised mean differences (SMDs) and 95\% confidence intervals were calculated for studies where there were sufficient data to calculate this using the Campbell Collaboration effect size calculator. ${ }^{20}$ We had planned to calculate odds ratios for dichotomous outcomes but found no study for which this was calculable. Negative SMDs indicate a reduction in stigma (benefit), i.e. an improvement in knowledge outcomes or a reduction in either negative attitudes or discriminatory behaviour in the intervention group. Where more than one outcome was reported within a category, the median effect size was presented. ${ }^{21}$ In the online tables we present data on the number of outcomes with statistically significant changes in outcome and the direction of effect to complement the effect size data of outcomes. ${ }^{21}$ These also provide some information about all included studies and at least some information on effectiveness for studies that reported insufficient data to calculate effect sizes. Owing to the considerable heterogeneity of the interventions, measures and participants in the included studies, it was not possible to conduct meta-analyses or to use conventional analytical methods to control for heterogeneity. As some studies had more than one intervention, this analysis was carried out at the intervention level with the number of participants in the control group split between the interventions, to control for unit of analysis error. ${ }^{22}$

We conducted two subgroup analyses on type of intervention by calculating, presenting and comparing median effect sizes attitude outcomes for each subgroup. The first analysis compared direct, indirect or no social contact, and the second compared target groups. We undertook similar sensitivity analyses to explore the possible effects of study design and risk of bias. First, we compared RCT evidence with non-RCT evidence, and second, within RCTs we compared the third of studies with the least risk of bias (see below) with the remainder.

\section{Assessment of study quality and risk of bias}

A quality assessment and profile of risk of bias within studies were carried out individually for all included studies. Level of RCT evidence was rated by two authors using the Cochrane risk of bias tool..$^{23}$ The third of RCTs with the lowest risk of bias are identified with an asterisk in the data extraction tables. To assess bias in non-randomised studies two researchers conducted quality appraisals using risk of bias criteria for non-randomised studies, ${ }^{23}$ suitable to the wide range of study designs included. When a decision about the risk of bias could not be made, it was resolved through discussion with a third author. In addition, for each study we indicated whether at least one outcome measure was validated, whether it was previously published, developed by the author or if items were used. Scales were marked as having evidence of psychometric adequacy providing they met one or more of the following 
criteria: the authors reported a Cronbach's $\alpha$ of 0.7 or greater, the authors referenced the measure as being reliable or valid, or there was some evidence of validity or reliability as judged by the review team.

\section{Results}

A total of 80 quantitative studies (422653 participants) were identified for inclusion in the review, 72 addressing research question 1 (long-term effectiveness) and 11 addressing research question 2 (setting), of which 3 studies addressed both questions (Fig. 1). The database search provided 27876 citations. After a review of the abstracts 26563 papers were excluded as they were clearly irrelevant or did not meet the inclusion criteria. The reference lists of 17 reviews were hand-checked and 49 further papers identified. Seven papers in press known to the authors were included. After removal of 330 duplicates the full text of the remaining 1061 potentially relevant papers was sought. Of these, 21 papers were unobtainable and 843 papers did not meet the inclusion criteria. Of the remaining papers 17 did not contain enough relevant data to extract. A full reference list is given in online Table DS2 and study characteristics are listed in online Tables DS3-5. Online Tables DS6 and DS7 give risk of bias and quality ratings for RCTs and other studies respectively.

\section{Medium- and long-term follow-up}

\section{Study characteristics}

Most of the studies addressing medium- or long-term outcomes took place in high-income countries (93\%), were aimed at school or university students (37\%) and used interventions comprising mental health education and literacy or mental health information $(43 \%)$. About a quarter $(28 \%)$ of the studies included were RCTs, $52 \%$ consisted of pre-post studies with or without a control group and $21 \%$ were longitudinal panel or cohort studies. Most studies (69\%) had a final follow-up assessment 1-6 months after the intervention had ended, whereas $21 \%$ had a longer follow-up (1-10 years post-intervention). Tables DS3 and DS4 show details of study characteristics.

\section{Evidence}

There were 72 quantitative studies with at least 4 weeks of follow-up, which included 81 interventions with 42653 participants. It was possible to calculate effect sizes and confidence intervals for 21 of these studies (23 interventions). These studies and their effect sizes are shown in Table 1. Findings based on statistical significance for all included studies are shown in Tables

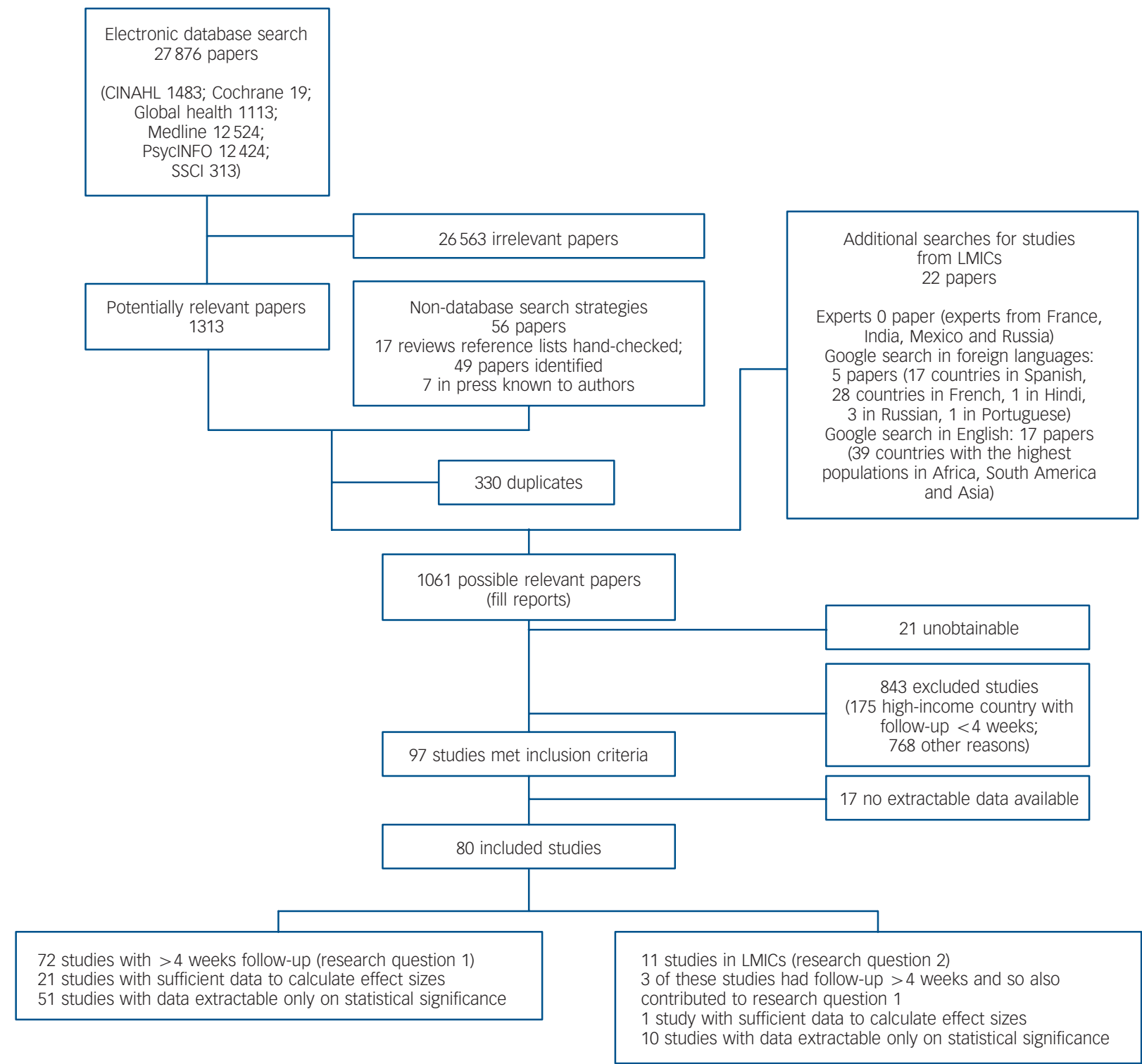

Fig. 1 Selection of papers and sources included in the review. LMIC, low- and middle-income country; SSCl, Social Science Citation Index 
DS3 (RCT, controlled and uncontrolled pre-post studies) and DS4 (longitudinal panel study or cohort design). For knowledge outcomes the median effect size was 0.54 indicating a medium effect in increasing knowledge. ${ }^{19}$ For attitude outcomes SMDs ranged from 0.05 to -1.22 with a median effect size of -0.26 , indicating a small reduction in stigmatising attitudes. For behavioural outcomes SMDs were calculated in one intervention which showed a small $(\mathrm{SMD}=0.22)$ effect in reducing stigmatising behaviour. Inspection of the pattern of significance findings for scales for all the included studies with medium- or long-term follow-up indicated that there were similar numbers of significant and non-significant findings indicating an increase in knowledge (26 v. 22). Similar numbers were also found for attitude scales (63 non-significant findings $v$. 52 significant in the direction of stigma reduction). Five scales had significant findings indicating an increase in stigma. For behavioural outcomes measured with scales, non-significant findings outnumbered significant ones indicating a reduction in discriminatory behaviour $(12 v .2)$ and this was also the case for behavioural outcomes measured at the item level (38v. 19).

Our subgroup analysis of type of intervention found that interventions containing direct social contact had a smaller median effect size for stigmatising attitudes $(-0.17)$ than those with indirect social contact $(-0.32)$ or no social contact $(-0.33)$. There were enough interventions with effect sizes to make comparisons of median effect sizes by three types of target group, and we found that interventions targeted at health professionals had a somewhat higher median effect size $(-0.41)$ than those targeting school pupils $(-0.21)$ or university students $(-0.13)$.

\section{Risk of bias}

Across all RCTs there was a low risk of bias for $50 \%$ of the criteria and an unclear or high risk of bias in the other $50 \%$. Only five trials met $70 \%$ or more of the criteria. Nine trials met between $40 \%$ and $60 \%$ of the criteria and five only met $15-30 \%$. In light of the nature of anti-stigma interventions it was not possible to mask participants and personnel to allocation, with the exception of one trial which was internet-based and thus easier to conceal. ${ }^{24}$ Of the 19 trials, 17 used at least one validated scale to measure outcomes, whereas 2 used non-validated scales that had been used in previously published papers. There were 53 non-randomised studies, 30 of which did not have a control group. Among studies with a control group, 6 were deemed to have a low risk of selection bias with regard to the comparability between the intervention and control groups. In 26 studies there was a high risk of attrition bias, where more than $20 \%$ of the sample were lost to follow-up and no intention to treat analysis was carried out. Possible confounders were considered and controlled for in only $28 \%$ of studies. As with the RCTs, masking of participants and personnel was not possible owing to the type of intervention. Among non-randomised studies, 24 had at least one validated outcome measure, 2 had at least one that was previously published, 4 had one that was specifically developed for the study with no psychometric testing reported, and 23 used items only. Details of risk of bias in individual studies are given in Tables DS5 and DS6. The median effect size for RCTs was lower than for nonrandomised controlled studies $(-0.17 v .-0.37)$. Within RCTs the third with the least risk of bias had a higher effect size $(-0.30)$ than the remainder $(-0.09)$.

\section{Evidence from LMICs}

There were 11 studies (1967 participants) from LMIC settings, 8 with less than a 4-week follow-up and 3 with longer follow-up. Study characteristics and statistical significance findings for these are shown in Table DS5. Eight of these were from upper middleincome countries and three were from lower middle-income countries. There was no study meeting our criteria from a lowincome country. Six studies were aimed at school and university students, two at caregivers of people with schizophrenia, and three at healthcare professionals. Three studies used an RCT design, one of which was a cluster randomised trial analysed within groups, two were controlled studies and six were uncontrolled pre-post studies. Within the 11 studies included there were 16 intervention arms, with 5 measuring knowledge outcomes and 14 measuring attitude outcomes. None of the studies had behavioural outcomes. Sufficient data to calculate an effect size were reported in only one of the studies; ${ }^{25}$ in this study - a psychoeducation programme for caregivers of patients with schizophrenia in Chile - the SMD for stigmatising attitudes was -2.11 (95\% CI -2.87 to -1.34 ), indicating a large effect. Inspection of the statistical significance of the knowledge scale findings for all studies revealed that both studies with such outcomes found no evidence of change; however, there were findings indicating a significant reduction in stigmatising attitudes for 11 of the 12 attitude scale outcomes assessed in these studies (Table DS5).

These results should be interpreted with caution. In seven of the studies, follow-up assessments were undertaken immediately after the intervention (in one study this was done 1 week after the intervention had ended). There were also issues regarding bias: owing to a lack of information in the papers it was generally difficult to gauge the extent of risk of bias. For the three RCTs, in $52 \%$ of criteria the risk of bias was unclear. Where information was provided, a high risk of bias was found in $19 \%$ of criteria across the RCTs, whereas in $29 \%$ of criteria the risk was low. This was most common for the incomplete outcome data and selective outcome reporting criteria. For the non-randomised studies, risk of bias varied across criteria, with $33 \%$ classified as high and $33 \%$ as low, and for $33 \%$ the degree of risk was unclear.

\section{Behavioural outcomes}

Among the 15 studies that did report behavioural outcomes, 7 assessed contact with someone with a mental health problem, 4 measured perceived discrimination and coping strategies in participants who had a mental health problem, 2 measured changes in school and workplace policies regarding mental health, ${ }^{26,27} 2$ measured experienced discrimination reported by people with mental health problems, ${ }^{28,29}$ and only 1 measured actual discriminatory behaviour by participants in the general population. ${ }^{30}$

\section{Discussion}

Our synthesis of 72 studies with follow-up beyond 4 weeks revealed that, at this follow-up, interventions aimed at reducing mental health-related stigma typically had a medium-sized effect on knowledge outcomes and a small effect on attitudinal outcomes, although for both types of outcome statistically nonsignificant findings were as common as significant ones. There were insufficient data on behavioural outcomes to draw any conclusions on the medium- or long-term effectiveness of interventions to reduce discrimination. This is the first systematic review to synthesise evidence on medium- and long-term effectiveness, which is striking given that stigma is often experienced by people with mental illness as a long-term difficulty. Although a number of systematic reviews indicated that social contact interventions were particularly effective, ${ }^{11,13,15,16}$ the majority of studies in these reviews had only short-term follow-up. Our review, restricted to studies with medium- and 
Table 1 Evidence for medium- and long-term effectiveness of interventions to reduce mental health-related stigma

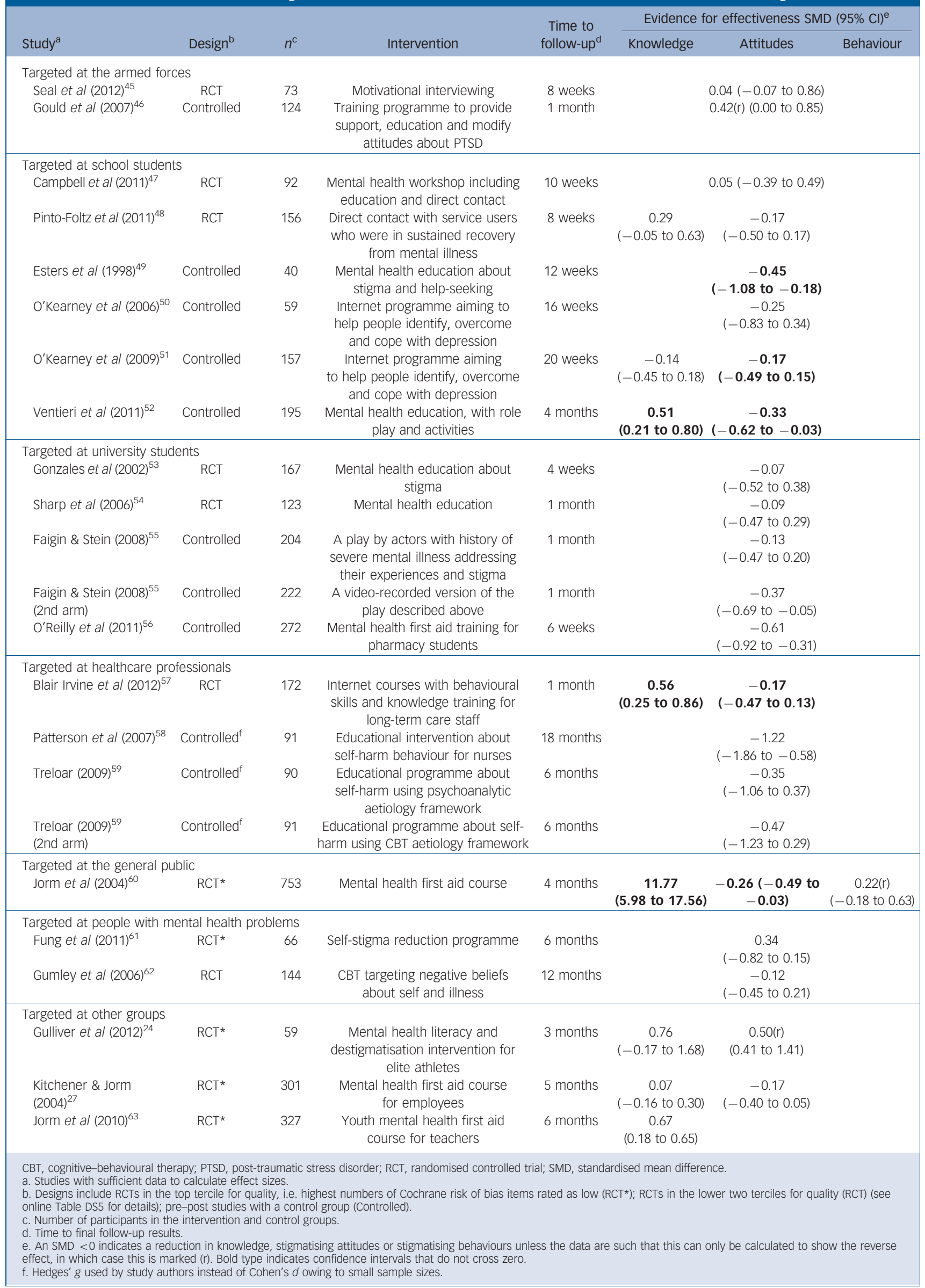


longer-term outcomes, did not support the superiority of social contact interventions as we had expected. As it is vital that stigma reduction is sustained in the longer term, the effectiveness of such social contact interventions clearly warrants further research.

Study quality was variable, and indeed study design and quality did appear to affect median effect sizes, although these subgroup and sensitivity analysis findings should be interpreted with caution owing to the heterogeneity of the studies. Overall, where we did identify positive changes from the interventions, the magnitude of the effects was generally rather modest. It is also clear that there is therefore a lack of research on actual discriminatory behaviour within the stigma research field.

For our second research question regarding LMICs, we found comparatively few studies from middle-income countries and none from low-income countries. A large effect size was found for the one LMIC study for which there were sufficient data to calculate the effect size and the majority of attitude scale outcomes indicated significant improvements in attitudes, although such findings must be treated with considerable caution. There is a clear need for more stigma reduction studies, particularly from low-income countries.

Our results regarding service user social contact are consistent with those of Griffiths et $a l^{31}$ who recently published a metaanalysis of RCTs of interventions intended to reduce stigma. Analysing data from 26 trials they found that interventions targeting personal stigma or social distance yielded small but significant reductions in stigma across all mental disorders. Further, they reported that educational interventions were effective in reducing personal stigma, as were interventions incorporating service user contact. This study also considered internet use and self-stigma and found that internet programmes were at least as effective in reducing personal stigma as face-toface delivery (see also Clement et al). ${ }^{32}$ They found no evidence that stigma interventions were effective in reducing self-stigma. In our review, although social contact appears to be the most strongly evidence-based type of intervention to reduce stigma when measured by immediate post-intervention outcomes, there is not at present evidence to show that such immediate benefits persist in the longer term.

\section{Limitations of the study}

This review has a number of limitations. In conducting a comprehensive overview of all relevant literature we have identified considerable heterogeneity among participant groups, interventions and outcomes. For example, we identified 55 different scales used for the 136 outcomes measured. Study quality also varied considerably. We were able to include studies in some non-English languages, but it is possible that we missed important projects published in other languages, for example potentially important studies not available at all in English, or studies for which only abstracts were available in English, and which we were not able to assess fully (see, for example, Shi et al). ${ }^{33}$ The temporal limitation of the search start date being 1980 will have resulted in the review missing studies before that date. We also need to acknowledge the possibility of publication bias, for example that intervention studies showing no difference might be published less often than those that do identify a clear benefit. Further, the risk of bias results given above, with half of all studies having a high or unknown risk of bias, mean that considerable caution needs to be exercised in interpreting these findings. It is also notable that relatively few of the interventions assessed following published, manualised procedures or including any rating of treatment fidelity. It should also be appreciated that although a narrative review may be able to disaggregate the nature of the interventions, and the specific target groups, into a greater number of specific subtypes, the numbers of studies in each of these categories would be small, and that this would give a greater descriptive richness at the expense of the wider generalisability of the findings. The systematic review method used here does not allow this narrower focus.

\section{Challenges in the measurement of stigma}

The assessment and validation of instruments to measure stigma and discrimination against people with mental illness has been under way since the 1960s. Although early measures such as the Opinions About Mental Illness and the Community Attitudes to Mental Illness scales are still used in some studies, ${ }^{34,35}$ there have been many developments in the breadth and quantity of measures to assess stigma in recent years. These include a trend to incorporate multiple outcomes or domains, for example knowledge and behaviour as well as attitudes; techniques to control for social desirability bias such as implicit measures; research on coping or 'stigma resilience'; and assessments among multiply stigmatised groups, such as people from ethnic minorities with mental illness. Despite these developments there are still substantial gaps in what can be assessed using available measures, including a lack of behavioural and structural indicators. We have seen in this review that behaviour is underrepresented in stigma intervention outcomes, for example changes in behaviour of others rated by patients or service users, or directly observed discrimination-related outcomes. There is a further gap in terms of important subgroups. For example, Link et al noted that children and adolescents were represented in only 3.7\% of stigma studies. ${ }^{36}$ More specific and tailored measures might facilitate inclusion of specific subpopulations in stigma research, such as those already affected by discrimination on the grounds of (for example) ethnicity. Additionally, studies that include measures validated in LMICs are rare, and only a few include any intervention component developed specifically in such countries. Future efforts should therefore address these gaps, because measurement and evaluation are critical to understanding the underlying mechanisms and effectiveness of anti-stigma interventions. A further challenge is to stop the use of unvalidated measures and item level analyses, while retaining enough flexibility to promote conceptual, contextual and theoretical relevance.

\section{Gaps in the evidence base}

This review has highlighted clear gaps in the field of anti-stigma interventions and research methods and a need for the harmonisation of outcomes in this field of research. These include the paucity of evidence on discrimination outcomes, or on reducing negative behaviours or increasing positive behaviours towards people with mental illness, ${ }^{37}$ and the lack of studies of specific target groups such as employers or family members, despite service users commonly reporting experiencing discrimination from both of these groups. ${ }^{38}$ There is an important need to assess whether benefits identified in the short term are maintained in the longer term, and if any booster interventions are needed to achieve sustainability. This review has also shown a relatively narrow focus of work to date: either on the general population (in attitude surveys) or on students within settings accessible to researchers (e.g. universities and colleges). ${ }^{16}$ From a global health viewpoint there is a distinct lack of interventional research in LMICs, despite emerging evidence of the scale and severity of the challenges posed by stigma and discrimination, and despite the fact that $85 \%$ of the world's population live in such countries. ${ }^{39,40}$ Finally, there is a need for more studies using high-quality research designs. Only a third of studies included in this paper used an RCT or other robust study design, and many of these had a high risk of bias. 


\section{Future research}

Knowledge in this field is generally from small studies of poor methodological quality, using inconsistent outcomes scales, and in particular few strong RCTs or interrupted time series studies have been carried out to test interventions intended to reduce stigma and discrimination. Our summary of previous systematic reviews does tend to support the view that social contact is the more effective type of intervention known to reduce stigma, at least in the short term. ${ }^{41}$ We do not yet have even weak consistent evidence to support interventions for target groups identified as priorities by service user groups, such as family members, and only an embryonic evidence base concerning how to address stigma in healthcare staff. ${ }^{42}$ Indeed, this degree of evidential neglect could itself be seen as a manifestation of structural discrimination. Given the magnitude of the challenges posed by stigma and discrimination, it is clear that there needs to be a commensurate concerted effort to fund methodologically strong research to provide robust evidence to support policy decisions on investment and interventions. Such a wider policy framework is now emerging. ${ }^{43}$ The World Health Organization Mental Health Action Plan, ratified by the World Health Assembly in May 2013, states as its vision:

'A world in which mental health is valued, promoted and protected, mental disorders are prevented and persons affected by these disorders are able to exercise the full range of human rights and to access high quality, culturally-appropriate health and social care in a timely way to promote recovery, in order to attain the highest possible level of health and participate fully in society and at work, free from stigmatization and discrimination.' ${ }^{44}$

Specifically, paragraph 75 of the Action Plan indicates a need to prioritise:

'Mental health promotion and prevention: provide technical support to countries on the selection, formulation and implementation of evidence-based and cost-effective best practices for promoting mental health, preventing mental disorders, reducing stigmatization and discrimination, and promoting human rights across the lifespan. ${ }^{\prime 44}$

This review indicates that an early necessity is to conduct more high-quality research to allow this policy priority to be firmly evidence-based, especially within LMICs.

\footnotetext{
Nisha Mehta, MBBS, Sarah Clement, PhD, Elena Marcus, MSc, Anne-Claire Stona, BSC, Nikita Bezborodovs, MD, Sara Evans-Lacko, PhD, Jorge Palacios, PhD, Mary Docherty, MRCPsych, Elizabeth Barley, PhD, Diana Rose, PhD, Mirja $\mathrm{PhD}$, Mary Docherty, MRCPsych, Elizabeth Barley, PhD, Diana Rose, PhD, Mirja
Koschorke, PhD, Health Service and Population Research Department, King's college London, Institute of Psychiatry, Psychology and Neuroscience, London, UK; Rahul Shidhaye, PhD, Centre for Mental Health, Public Health Foundation of India, Delhi, India; Claire Henderson, PhD, Graham Thornicroft, PhD, Health Service and Population Research Department, King's College London, Institute of Psychiatry, Psychology and Neuroscience' London, UK

Correspondence: Professor Graham Thornicroft, Health Service and Population Research Department, Box PO29, Institute of Psychiatry, Pschology and Neuroscience, De Crespigny Park, London SE5 8AF, UK. Email: graham.thornicroft@kcl.ac.uk

First received 20 Sep 2013, final revision 4 Jan 2015, accepted 27 Feb 2015
}

\section{Funding}

This publication is independent research funded by the National Institute for Health Research (NIHR) under its Programme Grants for Applied Research scheme (Improving Mental Health Outcomes by Reducing Stigma and Discrimination: RP-PG-0606-1053) (authors G.T., S.C., C.H., D.R.). This research was supported by the NIHR Collaboration
(a) for Leadership in Applied Health Research and Care South London at King's College London Foundation Trust. G.T. is supported by the European Union Seventh Framework Programme (FP7/2007-2013) Emerald project. This paper is an output of the PRogramme for Improving Mental health carE (PRIME). The material has been funded by aid from the UK Government; however, the views expressed do not necessarily reflect the UK Government's official policies. The research supported by the NIHR Collaboration for Leadership in Applied Health Research and Care South London at King's College London Leadership in Applied Health Research and Care South London at King's College London
Foundation Trust. G.T. is also funded in relation to the NIHR Specialist Mental Health Biomedical Research Centre at the Institute of Psychiatry, Psychology and Neuroscience, King's College London and the South London and Maudsley National Health Service (NHS) Foundation Trust. The views expressed in this publication are those of the authors and not necessarily those of the NHS, the NIHR or the Department of Health. M.K. was supported by a Wellcome Trust Clinical PhD Fellowship during her involvement in the review. N.B. was funded by an Erasmus grant from the European Commission. The work review. N.B. Was funded by an Erasmus grant from the European Commission. The work
leading to these results has received funding from the European Union Seventh Framework Programme (FP7/2007-2013) under grant agreement 305968.

\section{Acknowledgements}

We acknowledge our gratitude to the following colleagues for their contributions to this paper: Luigi de Benedictis and Dmitry Krupchanka (grey literature search); Nikhil Gupta (risk of bias assessments and referencing); Henry Lishi Li (extraction of one Chinese paper); and Shinsuke Koike (helped to extract one Japanese paper).

\section{Appendix}

\section{Eligibility criteria for study inclusion}

Participants

Any, except target populations that solely comprised people with dementia, substance misuse, intellectual disabilities or developmental disorders.

\section{Setting}

Any.

\section{Intervention}

Any intervention with a stated aim of changing mental health-related stigma, or with an implied aim of changing stigma as indicated by the inclusion of at least one of the following stigma-related outcomes: stigma (including internalised stigma), prejudice (attitudes and related outcomes), discrimination, or public mental health awareness/mental health literacy. Interventions relating to functional mental illnesses were included, those solely about dementia, substance misuse, learning disabilities or developmental disorders were excluded.

\section{Comparison}

Inactive or baseline comparator.

\section{Outcomes}

Outcomes comprising:

- knowledge

- attitudes (prejudice/self-attitudes)

- behaviour (discrimination/stigma-coping)

- follow-up at least 4 weeks after the intervention was completed (research question 1) or any (research question 2).

\section{Study design}

Any quantitative design.

\section{References}

1 Goffmann E. Stigma: Notes on the Management of Spoiled Identity. Prentice Hall, 1963.

2 Weiss MG, Ramakrishna J, Somma D. Health-related stigma: rethinking concepts and interventions. Psychol Health Med 2006; 11: 277-87.

3 Sartorius N, Schulze H. Reducing the Stigma of Mental Illness. Cambridge University Press, 2005.

4 Rabkin J. Public attitudes toward mental illness: a review of the literature. Schizophr Bull 1974; 10: 9-33.

5 Link BG, Phelan JC, Bresnahan M, Stueve A, Pescosolido BA. Public conceptions of mental illness: labels, causes, dangerousness, and social distance. Am J Public Health 1999; 89: 1328-33.

6 Thornicroft G. Shunned: Discrimination Against People with Mental IIIness. Oxford University Press, 2006.

7 Yang $\mathrm{LH}$, Cho SH, Kleinman A. Stigma of mental illness. In Mental and Neurological Public Health: A Global Perspective (ed. V Patel): 484-95. Elsevier, 2010.

8 Yang LH, Kleinman A, Link BG, Phelan JC, Lee S, Good B. Culture and stigma: adding moral experience to stigma theory. Soc Sci Med 2007; 64: 1524-35.

9 Raguram R, Raghu TM, Vounatsou P, Weiss MG. Schizophrenia and the cultural epidemiology of stigma in Bangalore, India. J Nerv Ment Dis 2004; 192: 734-44.

10 Weiss MG, Jadhav S, Raguram R, Vounatsou P, Littlewood R. Psychiatric stigma across cultures: local validation in Bangalore and London. Anthropol Med 2001; 8: 71-87.

11 Corrigan PW, Morris SB, Michaels PJ, Rafacz JD, Rusch N. Challenging the public stigma of mental illness: a meta-analysis of outcome studies. Psychiatr Serv 2012; 63: 963-73.

12 Mansouri N, Gharaee B, Shariat SV, Bolhari J, Nooraie RY, Rahimi-Movaghar A, et al. The change in attitude and knowledge of health care personnel and general population following trainings provided during integration of mental 
health in primary health care in Iran: a systematic review. Int J Ment Health Syst 2009; 3: 15

13 Clement S, Lassman F, Barley E, Evans-Lacko S, Williams P, Yamaguchi S, et al. Mass media interventions for reducing mental health-related stigma. Cochrane Database Syst Rev 2013; 7: CD009453.

14 Mittal D, Sullivan G, Chekuri L, Allee E, Corrigan PW. Empirical studies of selfstigma reduction strategies: a critical review of the literature. Psychiatr Serv 2012; 63: 974-81.

15 Holzinger A, Dietrich S, Heitmann S, Angermeyer M. Evaluation of targetgroup oriented interventions aimed at reducing the stigma surrounding mental illness. Psychiatr Prax 2008; 35: 376-86.

16 Yamaguchi S, Wu SI, Biswas M, Yate M, Aoki Y, Barley EA, et al. Effects of short-term interventions to reduce mental health-related stigma in university or college students: a systematic review. J Nerv Ment Dis 2013; 201: $490-503$.

17 Ando S, Clement S, Barley E, Thornicroft G. The simulation of hallucinations to reduce the stigma of schizophrenia: a systematic review. Schizophr Res 2011; 133: 8-16.

18 Schachter HM, Girardi A, Ly M, Lacroix D, Lumb AB, van Berkom J, et al. Effects of school-based interventions on mental health stigmatization: a systematic review. Child Adolesc Psychiatr Ment Health 2008; 2: 18.

19 Cohen J. Statistical Power Analysis for the Behavioral Sciences. Erlbaum, 1988

20 Wilson DB. Practical Meta-Analysis Effect Size Calculator. Campbell Collaboration, 2001 (http://www.campbellcollaboration.org/resources/ effect_size_input.php).

21 Grimshaw J, McAuley LM, Bero LA, Grilli R, Oxman AD, Ramsay C, et al. Systematic reviews of the effectiveness of quality improvement strategies and programmes. Qual Saf Health Care 2003; 12: 298-303.

22 Higgins JPT, Green S (eds). Cochrane Handbook for Systematic Reviews of Interventions version 5.0.2, ch 16.5. Cochrane Collaboration, 2009.

23 Higgins JP, Altman DG, Gotzsche PC, Juni P, Moher D, Oxman AD, et al. The Cochrane Collaboration's tool for assessing risk of bias in randomised trials. BMJ 2011; 343: 5928.

24 Gulliver A, Griffiths KM, Christensen H, Mackinnon A, Calear AL, Parsons A, et al. Internet-based interventions to promote mental health help-seeking in elite athletes: an exploratory randomized controlled trial. $J$ Med Internet Res 2012; 14: 120-37.

25 Gutierrez-Maldonado J, Caqueo-Urizar A, Ferrer-Garcia M. Effects of a psychoeducational intervention program on the attitudes and health perceptions of relatives of patients with schizophrenia. Soc Psychiatry Psychiatr Epidemiol 2009; 44: 343-8.

26 Jorm AF, Kitchener BA, Fischer JA, Cvetkovski S. Mental health first aid training by e-learning: a randomized controlled trial. Aust NZ J Psychiatry 2010; 44: 1072-81.

27 Kitchener BA, Jorm AF. Mental health first aid training in a workplace setting: a randomized controlled trial. BMC Psychiatry 2004; 4: 23.

28 Corker E, Hamilton S, Henderson C, Weeks C, Pinfold V, Rose D, et al. Experiences of discrimination among people using mental health services in England 2008-11. Br J Psychiatry 2013; 202 (suppl 55): s58-63.

29 Henderson C, Corker E, Lewis-Holmes E, Hamilton S, Flach C, Rose D, et al. England's time to change antistigma campaign: one-year outcomes of service user-rated experiences of discrimination. Psychiatr Serv 2012; 63: 451-7.

30 Evans-Lacko S, Henderson C, Thornicroft G. Public knowledge, attitudes and behaviour regarding people with mental illness in England 2009-2012. Br J Psychiatry 2013; 202 (suppl 55): s51-7.

31 Griffiths KM, Carron-Arthur B, Parsons A, Reid R. Effectiveness of programs for reducing the stigma associated with mental disorders. A meta-analysis of randomized controlled trials. World Psychiatry 2014; 13: 161-75.

32 Clement S, van Nieuwenhuizen A, Kassam A, Flach C, Lazarus A, de Castro M, et al. Filmed $v$. live social contact interventions to reduce stigma: randomised controlled trial. Br J Psychiatry 2012; 201: 57-64.

33 Shi ZY, Liu SY, Nie LY The impact of group psychological interventions on the stigma perceived of patients with depression. J Nurs Training 2012; 27 : 1213-1216.

34 Cohen J, Struening EL. Opinions about mental illness in the personnel of two large mental hospitals. J Abnorm Soc Psychol 1962; 64: 349-60.

35 Taylor SM, Dear MJ, Hall GB. Attitudes toward the mentally ill and reactions to mental health facilities. Soc Sci Med 1979; 13: 281-90.

36 Link BG, Yang LH, Phelan JC, Collins PY. Measuring mental illness stigma Schizophr Bull 2004; 30: 511-41.

37 Sharac J, Mccrone P, Clement S, Thornicroft G. The economic impact of mental health stigma and discrimination: a systematic review. Epidemiol Psichiatr SOC 2010; 19: 223-32.

38 Henderson $C$, Williams $P$, Little $K$, Thornicroft $G$. Mental health problems in the workplace: changes in employers' knowledge, attitudes and practices in England 2006-2010. Br J Psychiatry 2013; 202 (suppl 55): s70-6.
39 Thornicroft G, Brohan E, Rose D, Sartorius N, Leese M. Global pattern of experienced and anticipated discrimination against people with schizophrenia: a cross-sectional survey. Lancet 2009; 373: 408-15.

40 Lasalvia A, Van Weeghel J, Reneses B, Bacle SV, Thornicroft G, Bonetto C. Discrimination reported by people with major depressive disorder: authors reply. Lancet 2013; 381: 1181-2.

41 Henderson $\mathrm{C}$, Thornicroft G. Evaluation of the Time to Change programme in England 2008-2011. Br J Psychiatry 2013; 202 (suppl 55): s45-8.

42 Jones S, Howard L, Thornicroft G. 'Diagnostic overshadowing': worse physical health care for people with mental illness. Acta Psychiatr Scand 2008; 118: 169-71.

43 United Nations. Convention on the Rights of Persons with Disabilities. UN, 2006.

44 World Health Organization. Global Mental Health Action Plan 2013-2020. WHO, 2013.

45 Seal KH, Abadjian L, McCamish N, Shi Y, Tarasovsky G, Weingardt K. A randomized controlled trial of telephone motivational interviewing to enhance mental health treatment engagement in Iraq and Afghanistan veterans. Gen Hosp Psychiatry 2012; 34: 450-9.

46 Gould M, Greenberg N, Hetherton J. Stigma and the military: evaluation of a PTSD psychoeducational program. J Trauma Stress 2007; 20: 505-15.

47 Campbell M, Shryane N, Byrne R, Morrison AP. A mental health promotion approach to reducing discrimination about psychosis in teenagers. Psychosis 2011; 3: 41-51.

48 Pinto-Foltz MD, Logsdon MC, Myers JA. Feasibility, acceptability, and initial efficacy of a knowledge-contact program to reduce mental illness stigma and improve mental health literacy in adolescents. Soc Sci Med 2011; 72: 2011-9.

49 Esters IG, Cooker PG, Ittenbach RF. Effects of a unit of instruction in mental health on rural adolescents' conceptions of mental illness and attitudes about seeking help. Adolescence 1998; 33: 469-76.

50 O'Kearney R, Gibson M, Christensen H, Griffiths KM. Effects of a cognitivebehavioural internet program on depression, vulnerability to depression and stigma in adolescent males: a school-based controlled trial. Cogn Behav Ther 2006; 35: 43-54.

51 O'Kearney R, Kang K, Christensen H, Griffiths K. A controlled trial of a schoolbased Internet program for reducing depressive symptoms in adolescent girls. Depress Anxiety 2009; 26: 65-72.

52 Ventieri D, Clarke DM, Hay M. The effects of a school-based educational intervention on pre-adolescents' knowledge of and attitudes towards mental illness. Adv Sch Ment Health Promot 2011; 4: 5-17.

53 Gonzales JM, Tinsley HEA, Krauder KR. Effects of psychoeducational interventions on opinions of mental illness, attitudes towards help seeking, and expectations about psychotherapy in college students. J Coll Stud Dev 2002; 43: 51-63.

54 Sharp W, Hargrove DS, Johnson L, Deal WP. Mental health education: an evaluation of a classroom based strategy to modify help seeking for mental health problems. J Coll Stud Dev 2006; 47: 419-38.

55 Faigin DA, Stein $\mathrm{CH}$. Comparing the effects of live and video-taped theatrical performance in decreasing stigmatization of people with serious mental illness. J Ment Health 2008; 17: 594-606.

56 O'Reilly CL, Bell JS, Kelly PJ, Chen TF. Impact of mental health first aid training on pharmacy students' knowledge, attitudes and self-reported behaviours: a controlled trial. Aust NZ J Psychiatry 2011; 45: 549-57.

57 Blair Irvine A, Billow MB, Eberhage MG, Seeley JR, McMahon E, Bourgeois M. Mental illness training for licensed staff in long-term care. Issues Ment Health Nurs 2012; 33: 181-94.

58 Patterson P, Whittington R, Bogg J. Testing the effectiveness of an educational intervention aimed at changing attitudes to self-harm. J Psychiatr Ment Health Nurs 2007; 14: 100-5.

59 Treloar AJ. Effectiveness of education programs in changing clinicians' attitudes toward treating borderline personality disorder. Psychiatr Serv 2009; 60: 1128-31.

60 Jorm AF, Kitchener BA, O'Kearney R, Dear KB. Mental health first aid training of the public in a rural area: a cluster randomized trial. BMC PSychiatry 2004; $4: 33$.

61 Fung KMT, Tsang HWH, Cheung WM. Randomized controlled trial of the self-stigma reduction program among individuals with schizophrenia. Psychiatr Res 2011; 189: 208-14.

62 Gumley A, Karatzias A, Power K, Reilly J, McNay L, O'Grady M. Early intervention for relapse in schizophrenia: impact of cognitive behavioural therapy on negative beliefs about psychosis and self-esteem. $\mathrm{Br} J \mathrm{Clin}$ Psychol 2006; 45: 247-60.

63 Jorm AF, Kitchener BA, Sawyer MG, Scales H, Cvetkovski S. Mental health first aid training for high school teachers: a cluster randomized trial. $B M C$ Psychiatry 2010; 10: 51.

कि EXTRA EXTRA
CONTEN
ONLINE 